\title{
Surgical Treatment for Kommerell's Diverticulum Associated with a Right-Sided Aortic Arch and an Aberrant Left Subclavian Artery: Endovascular or Hybrid
}

\author{
Atsushi Morishita, MD, ${ }^{1}$ Hideyuki Tomioka, MD, ${ }^{2}$ Seiichiro Katahira, MD, ${ }^{3}$ \\ Takeshi Hoshino, MD, ${ }^{4}$ and Kazuhiko Hanzawa, MD ${ }^{5}$
}

A right-sided aortic arch, associated with an aberrant left subclavian artery and a Kommerell's diverticulum, is a rare congenital anomaly. Case 1: A 53-year-old man, complaining of dysphasia, underwent a two-stage hybrid operation. Total arch replacement with the reconstruction of supraaortic vessels was performed via a median sternotomy. Thoracic endovascular aortic repair was subsequently completed with the femoral approach. Case 2: A 81-year-old man, complaining of syncope and dizziness, underwent thoracic endovascular aortic repair after endovascular aneurysm repair for a common iliac artery aneurysm. Treatment strategies for Kommerell's diverticulum should be individually determined depending on the clinical situation and anatomical features.

Keywords: Kommerell's diverticulum, thoracic endovascular aortic repair, two-stage hybrid operation

${ }^{1}$ Department of Cardiovascular Surgery, Numata Neurosurgery Heart-Disease Hospital, Numata, Gunma, Japan

${ }^{2}$ Department of Cardiovascular Surgery, Tokyo Women's Medical University Yachio Medical Center, Yachio, Chiba, Japan ${ }^{3}$ Department of Surgery, Tokyo Rosai Hospital, Tokyo, Japan ${ }^{4}$ Department of Anesthesiology, Minami Machida Hospital, Machida, Tokyo, Japan

${ }^{5}$ Department of Advanced Treatment and Prevention for Vascular Disease and Embolism, Niigata University Graduate School of Medical and Dental Sciences, Niigata, Niigata, Japan

Received: December 25, 2018; Accepted: January 27, 2019 Corresponding author: Atsushi Morishita, MD. Department of Cardiovascular Surgery, Numata Neurosurgery Heart-Disease Hospital, 8 Sakae-cho, Numata, Gunma 378-0014, Japan

Tel: +81-278-22-5052, Fax: +81-278-22-5469

E-mail: atsumori@samba.ocn.ne.jp

(cc) BY-NC-SA (02019 The Editorial Committee of Annals of Vascular Diseases. This article is distributed under the terms of the Creative Commons Attribution License, which permits use, distribution, and reproduction in any medium, provided the credit of the original work, a link to the license, and indication of any change are properly given, and the original work is not used for commercial purposes. Remixed or transformed contributions must be distributed under the same license as the original.

\section{Introduction}

A right-sided aortic arch (RAA), associated with an aberrant left subclavian artery (ALSA) and a Kommerell's diverticulum (KD), is a rare congenital anomaly. Surgical management is crucial before rupture or dissection occurs. Although the outcomes of the traditional open surgery have been favorable, the invasiveness of this treatment remains high. We report two cases of KD treated with endovascular and hybrid approaches.

\section{Case Report}

\section{Case 1}

A 53-year-old man presenting with chronic dysphasia and chest pain was admitted to our hospital. He had been treated with an unidentified complaint by a physician. Upon admission, his blood pressure was $128 / 62 \mathrm{mmHg}$ symmetrically. His pulse was regular (72 beats/min). Neither carotid bruit nor cardiac murmur was reported. Lung examination revealed clear breath sounds. Laboratory evaluations did not show any abnormalities. Transthoracic echocardiography (TTE) revealed normal left ventricular function unassociated with congenital heart disease. Computed tomography angiography (CTA) demonstrated an RAA and an ALSA originating from a KD (Fig. 1A). Cross-sectional mesurements of the KD revealed that the diameter of the diverticulum from the opposite aortic wall to the tip of that was $52 \mathrm{~mm}$, and the diameter at the origin of the ALSA from the aortic arch was $27 \mathrm{~mm}$. The aortic arch vessels arose in the following order: left common carotid artery, right common carotid artery, right subclavian artery, and ALSA. The fourth branch posteriorly passed to the esophagus from a bulbous diverticulum across the midline toward the left and upward. The left vertebral artery arose from the left common carotid artery. The patient was considered to be unsuitable for thoracic endovascular aortic repair (TEVAR) alone because 


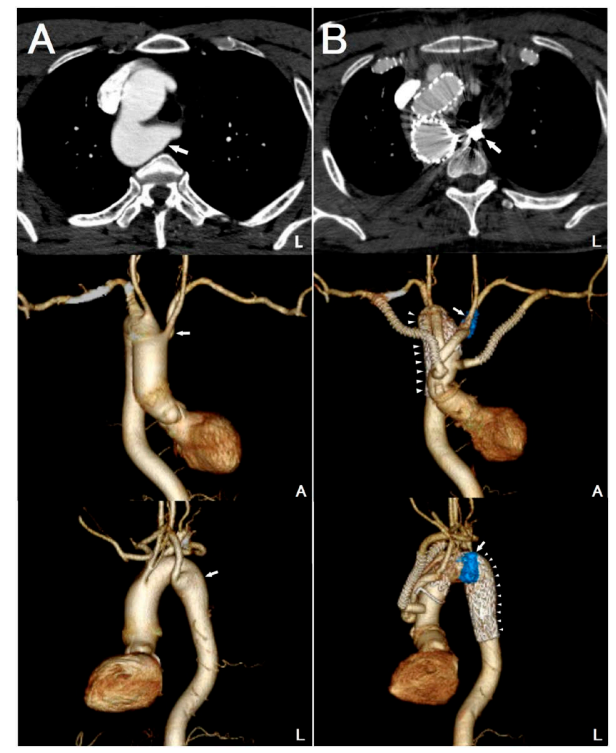

Fig. 1 Computed tomography (CT) scan in Case 1.

(A) Preoperative CT showing a right-sided aortic arch and an aberrant left subclavian artery originating from the Kommerell's diverticulum (KD; white arrow). (B) Postoperative CT showing good patency of the bypass grafts to the supra-aortic vessels and the complete exclusion of the KD by the stent-grafts (white arrowheads) and coils (white arrow).

A: anterior; L: left

of endoleak with insufficient proximal landing zone of stent-graft. We decided to perform two-stage surgery with endovascular treatment after written informed consent was obtained.

To ensure revascularization of the bilateral upper extremities, the bilateral axillary arteries were first prepared to be anastomosed with an 8 - $\mathrm{mm}$ ringed polytetrafluoroethylene graft. Through a median sternotomy, cardiopulmonary bypass was instituted in standard fashion with ascending aortic and bicaval cannulation. The KD and an adjoining ALSA in the surgical field was difficult to confirm. Under deep hypothermic circulatory arrest, both common carotid arteries were reconstructed using the arch-first technique with retrograde cerebral perfusion. The orifice of the right subclavian artery was doubly ligated. Antegrade cerebral perfusion was started through a four-branch Dacron graft. Distal anastomosis, located on the right side of the trachea, was created just behind the right common carotid artery without an elephant trunk. Subsequently, the tube graft anastomosed at the distal side was combined with the main graft to achieve cerebral perfusion using a stepwise technique. Resumption of the whole body was performed, and proximal anastomosis was achieved with the transected aorta just above the sino-tubular junction. According to the reconstruction between graft conduits anastomosed to the bilateral axillary arteries and the side branches of the four-branch Dacron graft retrieved through the thorax, total arch replacement was completed. The postoperative hemodynamics state was stable. The artificial conduit competed with the native left subclavian artery with regard to blood flow. Because there was a concern that the graft anastomosed to the left axillary artery might be occluded by a thrombus, an endovascular procedure was performed the next day. The time interval between prior total arch replacement and TEVAR was $16 \mathrm{~h}$. The right femoral artery was exposed through an oblique incision. A $31 \times 150-\mathrm{mm}$ conformable GORE TAG stent-graft (W. L. Gore and Associates, Inc., Flagstaff, AZ, USA) was advanced in retrograde fashion across the angulated aortic arch and subsequently deployed just distal to the side branch of the four-branch Dacron graft that controlled the perfusion to the right arm. Because endoleak was detected using angiography, the $31 \times 100-\mathrm{mm}$ conformable GORE TAG stent-graft was additionally inserted to ensure the coverage of the ostium of the KD. The left brachial artery was percutaneously cannulated with a 6Fr introducer (Destination, Terumo, Tokyo, Japan). For the purpose of resolving dysphagia, we did not select means to completely occupy the KD by coils. To completely exclude the $\mathrm{KD}$, the non-dilated origin of the ALSA was intentionally embolized with detachable coils (MICRUSFRAME; Johnson \& Johnson, New Brunswick, NJ, USA) to accurately construct the frame at an early stage. Further, the space in the frame was packed with pushable coils (Tornade Embolization Coil; Cook, Bloomington, IN, USA) to reinforce the embolic effect. The completion angiography demonstrated a complete exclusion of the diverticulum. The postoperative course was uneventful without cerebrovascular failure, respiratory failure, or paraplegia. Postoperative CTA demonstrated good patency of the bypass grafts to the supra-aortic vessels, and complete exclusion of the KD by the stent-grafts and coils (Fig. 1B). The patient's dysphagia resolved, and he is being followed up for medical treatment once a month after discharge from the hospital.

\section{Case 2}

An 81-year-old man complaining of syncope and dizziness was admitted to our hospital. He had been treated for brain infarction and hereditary tyrosinemia under adequate anticoagulation therapy with warfarin. At admission, his blood pressure was $120 / 70 \mathrm{mmHg}$ symmetrically. His pulse rate was regular ( 58 beats/min). Laboratory evaluation showed no abnormalities. TTE revealed normal left ventricular function and moderate mitral regurgitation. CTA demonstrated a RAA, an ALSA originating from the $\mathrm{KD}$, and a saccular left common iliac artery aneurysm (Fig. 2A). Endovascular aneurysm repair (EVAR) for a saccular left common iliac artery aneurysm 


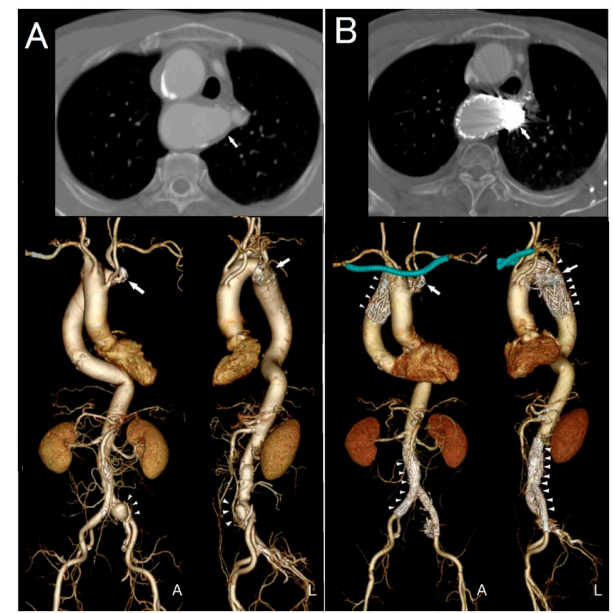

Fig. 2 Computed tomography (CT) scan in Case 2.

(A) Preoperative CT showing a right-sided aortic arch, an aberrant left subclavian artery originating from the Kommerell's diverticulum (KD; white arrow), and a saccular left common iliac artery aneurysm (white arrowheads). (B) Postoperative CT showing good patency of the bypass grafts to the left subclavian artery, complete exclusion of the KD and a saccular left common iliac artery aneurysm by the stent-grafts (white arrowheads) and coils (white arrow).

A: anterior; L: left

was performed in combination with coil embolization to a left internal iliac artery. Seven months after EVAR, follow-up computed tomography showed an enlargement of the KD. Cross-sectional measurement of the KD in detail revealed that the diameter from the opposite aortic wall to its tip was $73 \mathrm{~mm}$ and the diameter at the origin of the aberrant subclavian artery from the aortic arch was $40 \mathrm{~mm}$. Considering his medical history, the patient was deemed unsuitable for open surgery. The time interval between prior abdominal aortic aneurysm (AAA) surgery and TEVAR was 8 months. Because the proximal landing zone of the stent-graft above the KD was $2 \mathrm{~cm}$ in length, we decided to perform TEVAR after written informed consent was obtained.

To ensure revascularization to a left upper extremity after obliterating the $\mathrm{KD}$, the bypass with a $7-\mathrm{mm}$ ringed polytetrafluoroethylene graft was first prepared between the bilateral axillary arteries prior to TEVAR. The right femoral artery was exposed through an oblique incision. A $45 \times 100$-mm conformable GORE TAG stent-graft was deployed in retrograde fashion at the foundation of the upper descending aorta. Subsequently, the $45 \times 100-\mathrm{mm}$ conformable GORE TAG stent-graft was additionally inserted just distal to the right subclavian artery across the angulated aortic arch under right ventricular rapid pacing. To avoid the migration of the stent-graft, the touch-up via inflation of a balloon (tri-lobe balloon catheter, W. L. Gore and Associates, Inc., Flagstaff, AZ, USA) was per- formed only at the proximal side of the stent-graft. The left brachial artery percutaneously was cannulated with a 6Fr introducer. The left vertebral artery arose from the origin of the left subclavian artery adjacent to the KD. For the purpose of completely excluding the $\mathrm{KD}$, we had to completely occupy the KD using detachable and pushable coils. Angiography revealed no endoleak. The completion angiography demonstrated a complete exclusion of the diverticulum. The postoperative course was uneventful without cerebrovascular failure, respiratory failure, or paraplegia. Postoperative CTA demonstrated good patency of the bypass grafts to the left subclavian artery, complete exclusion of $\mathrm{KD}$, and the saccular left common iliac artery aneurysm by the stent-grafts and coils (Fig. 2B). The patient is being followed up for medical treatment once a month after discharge from the hospital. The patient was emergently admitted 21 months after TEVAR with syncope. Because electrocardiogram revealed complete atrio-ventricular block, a permanent pacemaker was implanted. The patient was transferred to the rehabilitation department of the hospital for disuse syndrome.

\section{Discussion}

$\mathrm{KD}$ is an outpouching aneurysm originating from aberrant subclavian arteries from the descending thoracic aorta. Embryologically, it is thought to be a remnant of the fourth primitive dorsal arch that does not regress. An RAA occurs in approximately $0.1 \%$ of the population. ${ }^{1)}$ Half of the cases of RAA are associated with an ALSA. The left ligamentum arteriosus usually joins the root of the ALSA to the left pulmonary artery. RAA with an ALSA and a left ductus arteriosus sometimes forms a vascular ring. This type of RAA is seldom associated with congenital heart disease. Because this anomaly usually does not significantly compress the trachea and esophagus, it is mostly unnoticed or manifests as non-specific symptoms until adulthood. Clinical symptoms associated with the appearance of the $\mathrm{KD}$, including dysphagia, wheezing, strider, shortness of breath, and chest pain, could be caused by the compression of neighboring structures as well as atherosclerotic changes of anomalous vessels. In a review of the literature concerning aneurysms arising in an aberrant subclavian artery, Austin and Wolfe reported that 19\% of affected patients died from ruptures. ${ }^{2)}$ Kouchoukos and Masetti reported that $20 \%$ of affected patients were associated with dissection. ${ }^{3)}$ In a review of the literature concerning RAA with an aneurysm of ALSA, Cinà et al. reported that the incidence of either rupture or dissection was $53 \%$ in affected patients. ${ }^{4)}$ Fisher et al. reported a case of a ruptured aneurysm arising from a right aberrant subclavian artery; the diameter at the orifice of the diverticulum measured only $2.0 \mathrm{~cm} .{ }^{5)}$ Because the entity is 
rare and there is limited data, it is thought to be difficult to predict the rupture of these aneurysms in terms of a parameter of their sizes alone. Further, Kim et al. reported that pathologic findings in resected KD specimens show mucoid medial degeneration related to atherosclerotic changes. ${ }^{6}$ Therefore, given the higher incidence of rupture or dissection and characteristic pathological findings, it would appear appropriate to recommend early surgical management even in asymptomatic patients.

Several options for surgical, endovascular, or hybrid techniques have been preferred as treatments for KD. However, there has been no established treatment strategy because of the rarity of the disease. Open surgical treatment has been described, including interposition grafts or endoaneurysmorrhaphy through thoracotomy with a left subclavian-to-carotid transposition, graft replacement with in situ reconstruction of the aberrant subclavian artery through thoracotomy, and total arch replacement through a median sternotomy and thoracotomy. Open repair of these aneurysms has a high mortality, ranging from $16 \%$ to $18 \% .^{2)}$ Kouchoukos and Masetti recently reported no hospital deaths with the standard surgical technique. ${ }^{3)}$ Furthermore, Tsukui et al. reported no hospital deaths with total arch replacement and anatomical reconstruction of the subclavian artery without endovascular technique. ${ }^{7)}$ Idrees et al. reported no hospital mortality in hybrid repair with endovascular technique. ${ }^{8)}$ Cinà et al. suggested aggressive treatment for aneurysms $\geq 3 \mathrm{~cm}$ in diameter. $\left.{ }^{4}\right)$ Ota et al. suggested that the diameter of aneurysms $>50 \mathrm{~mm}$ in symptomatic patients are indications for surgery. ${ }^{9}{ }^{9}$ Idrees et al. recommended surgery if the diameter as cross-sectionally measured from the opposite aortic wall to the tip of KD is at least $5 \mathrm{~cm}$, and the diameter as cross-sectionally measured at the origin of the aberrant subclavian artery from the aortic arch is at least $3 \mathrm{~cm} .{ }^{8)}$

Endovascular treatment for the KD is a less invasive approach than open surgery, which requires a thoracotomy or a median sternotomy. Indications for endovascular treatment should be considered with respect to clinical situations and anatomical factors. The former involves clinical symptoms, hemodynamic state, comorbidities, and past medical history. The latter is related to the characteristic configuration of a sharply-curved distal arch, a distorted descending aorta, and anomalous supra-aortic branches. Total endovascular repair with a branched device has been reported to further reduce the invasiveness of treatment. Reconstruction of the left subclavian artery would be of utmost importance to prevent arm claudication in younger patients, subclavian steal syndrome in the elderly, and vertebrobasilar insufficiency. However, this remains controversial.

In Case 1, because there was no distance between the right subclavian artery and the top of the sharply-curved distal arch, the endovascular approach was not feasible because of the greater occurrence of endoleaks and migration. A thoracotomy provides satisfactory exposure of the ascending aorta, transverse arch, and descending thoracic aorta. However, this approach may give rise to lung injury by thoracotomy and brain infarction with difficulty in air removal during hypothermic circulatory arrest. At our hospital, the 30-day postoperative mortality rate of the 31 patients who underwent the aortic arch surgery between January 2014 and December 2018 was 3.2\%. We adopted a hybrid repair, including initial total arch replacement without elephant trunk and subsequent endovascular exclusion of the KD with coil embolization of the left subclavian artery. Especially, when the graft inserted into the sharply-curved distal arch as an elephant trunk is transformed, it is anticipated to be difficult to deliver the stentgraft to its intended position in the subsequent TEVAR. Securing the distance between the back-most branch of the four-branch Dacron graft and the distal anastomosed site is crucial. We did not use the frozen elephant trunk because there was a possibility of an unexpected occurrence of paraplegia. The stent frame was designed as a stentgraft used as a frozen elephant trunk for the inner side and a stent-graft in TEVAR for the outer side. Endoleak might occur when the compatibility of overlapping varying characteristic stent-grafts is poor. There is a possibility of recurrent dysphagia because of tracheal and esophageal compression by the retained $\mathrm{KD}$, so close follow-up of endoleak of the stent-graft and recurrent symptoms by the retained $\mathrm{KD}$ would be required in the future.

In Case 2, because the patient underwent EVAR with coil embolization to the left internal iliac artery before 8 months, it was predicted to increase the risk of spinal cord ischemia after TEVAR. Schlösser et al. reported that prior infrarenal AAA repair is associated with increased risk of spinal cord ischemia after TEVAR compared with patients without prior AAA surgery. ${ }^{10)}$ It was mandatory to perform preventive management during TEVAR, including deployment of stent-grafts to make the length of aortic coverage as short as possible, maintaining the collateral network supplied from the covered left subclavian artery with revascularization, and meticulous attention to avoid intra-operative hypotension. Furthermore, we had to take care to deploy the stent-graft in a bird beak configuration on the lesser curvature of the arch. To strictly deploy stentgrafts, we usually perform right ventricular rapid pacing, when the proximal landing of the thoracic stent-graft is located in the range of zone 0 or zone 1 and supra-aortic branches anomalously exist. Cerebrospinal fluid drainage was omitted because of anticoagulation therapy. The technique, which bunched the next stent-graft upward toward the stent-graft deployed at the upper descending aorta as a 
foundation, was thought to be useful to avoid the migration of the stent-graft with high accuracy.

\section{Conclusion}

Although the technical advancement of stent-grafts and the development of treatment strategies have been remarkable, long-term outcomes of endovascular or hybrid treatment for the KD are uncertain. Our findings in the two cases indicate that to ensure optimal treatment outcomes, it is mandatory to individually evaluate current treatment strategies in accordance with clinical symptoms, hemodynamic state, comorbidities, past medical history, and anatomical features of KD.

\section{Disclosure Statement}

All authors have no conflict of interest.

\section{Author Contributions}

Study conception: AM

Writing: AM

Critical review and revision: all authors

Final approval of the article: all authors

Accountability for all aspects of the work: all authors

\section{References}

1) Hastreiter AR, D'Cruz IA, Cantez T. Right-sided aorta part I: occurrence of right aortic arch in various types of congenital heart disease. Br Heart J 1966; 28: 722-5.

2) Austin EH, Wolfe WG. Aneurysm of aberrant subclavian artery with a review of the literature. J Vasc Surg 1985; 2: 571-7.

3) Kouchoukos NT, Masetti P. Aberrant subclavian artery and Kommerell aneurysm: surgical treatment with a standard approach. J Thorac Cardiovasc Surg 2007; 133: 888-92.

4) Cinà CS, Althani $\mathrm{H}$, Pasenau J, et al. Kommerell's diverticulum and right-sided aortic arch: a cohort study and review of the literature. J Vasc Surg 2004; 39: 131-9.

5) Fisher RG, Whigham CJ, Trinh C. Diverticula of Kommerell and aberrant subclavian arteries complicated by aneurysms. Cardiovasc Intervent Radiol 2005; 28: 553-60.

6) Kim KM, Cambria RP, Isselbacher EM, et al. Contemporary surgical approaches and outcomes in adults with Kommerell diverticulum. Ann Thorac Surg 2014; 98: 1347-54.

7) Tsukui H, Aomi S, Yamazaki K. Surgical strategy for Kommerell's diverticulum: Total arch replacement. J Thorac Cardiovasc Surg 2014; 148: 1423-7.

8) Idrees J, Keshavamurthy S, Subramanian S, et al. Hybrid repair of Kommerell diverticulum. J Thorac Cardiovasc Surg 2014; 147: 973-6.

9) Ota T, Okada K, Takanashi S, et al. Surgical treatment for Kommerell's diverticulum. J Thorac Cardiovasc Surg 2006; 131: 574-8.

10) Schlösser FJV, Verhagen HJM, Lin PH, et al. TEVAR following prior abdominal aortic aneurysm surgery: increased risk of neurological deficit. J Vasc Surg 2009; 49: 308-14; discussion, 314. 